\title{
EDITORIAL
}

\section{Poor health literacy: a ‘hidden' risk factor}

"Much learning does not teach understanding." Heraclitus (540-480 BC)

$\mathrm{T}$ he study of health literacy among patients is a fairly new area of research, which has seen a surge in activity over the past decade. With this increasing body of literature, the health-care community is beginning to appreciate the magnitude of effect that poor literacy has on patient outcomes. A timely report in JAMA entitled "Can this patient read and understand written health information" highlights this important topic, providing the impetus for a closer look at the issues involved and how they might affect patients with cardiovascular disease (Powers, B. J. et al. JAMA 304, 76-84; 2010).

Health literacy has been defined as "the degree to which individuals have the capacity to obtain, process, and understand basic health information and services needed to make appropriate health decisions" (Selden, C. R. et al. National Library of Medicine, Bethesda, MD [online], http://www.nlm.nih.gov/ archive/20061214/pubs/cbm/hliteracy.pdf; 2000). In the early 1990s, a survey conducted in the US indicated that over 90 million adults had less than adequate health literacy skills, and that $20 \%$ of the adult population had a reading age of $10-11$ years or younger. More recently, a systematic review demonstrated that poor health literacy was consistently associated with patient age, ethnicity, and level of education (Paasche-Orlow, M. K. et al. J. Gen. Intern. Med. 20, 175-184; 2005). Low health literacy is particularly common among older people ( $>60$ years), who are also the age group most likely to be affected by chronic conditions such as cardiovascular disease. Importantly, however, much of the health literature given to patients-including information about their condition, hospital discharge instructions, patient-consent forms, and even medication bottles-is written at a high level of literacy. Understanding and acting upon this information often requires reading and comprehension skills at least equivalent to those expected of an $11^{\text {th }}$ grade high-school student. Clearly, a disparity exists.

Low health literacy has been associated with nonadherence to treatment plans and medical regimens, poor patient self-care, high health-care costs, and increased risk of hospitalization and mortality. Realizing that health literacy affects prognosis affords the opportunity to better understand the causes of poor outcome and develop interventions to address this issue. Many cardiovascular diseases have complex mechanisms and etiologies and can be difficult for patients to understand. Low health literacy, therefore, presents a particular challenge in treating the cardiac patient.

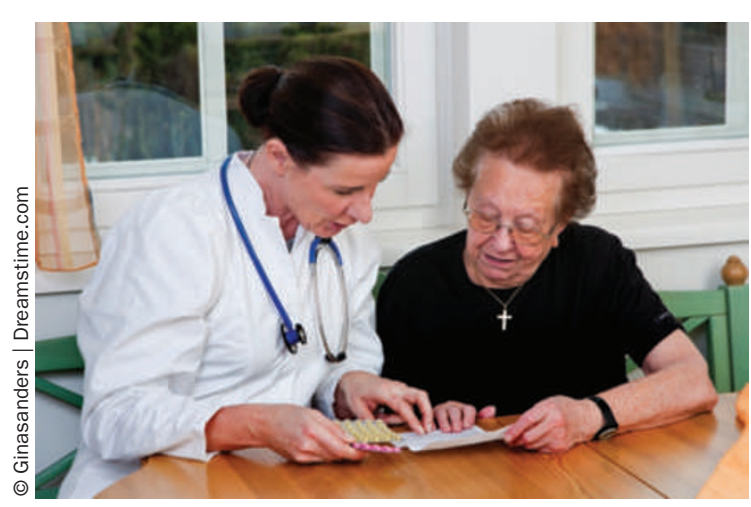

Part of the problem is that low health literacy is underrecognized. Sadly, patients are often too ashamed to tell their doctor that they cannot adequately read or understand the information given to them. Physicians must, therefore, become adept at detecting more subtle indicators that a patient has trouble reading, such as consistently 'forgetting' their reading glasses or deferring to a companion to whom they give written health information during the office visit.

Several tools do exist to help the physician identify patients with poor health literacy. The Rapid Estimate of Adult Literacy in Medicine, Revised (REALM-R) test and the Test of Functional Health Literacy in Adults (TOFHLA) are two such examples. These tests use word and number recognition, and can be performed in less than $10 \mathrm{~min}$. Although identifying patients with low literacy could help improve outcomes, 'blanket screening' with tests such as REALM-R and TOFHLA is controversial. One school of thought is that clearly written health-care information, which is easy to understand, should be provided to every patient not just those with poor literacy.

The UK National Health Service has published guidelines for improving the quality and readability of written information given to patients (NHS Institute for Innovation and Improvement [online], http://www.institute.nhs.uk/ quality and service improvement tools/quality and service_improvement_tools/patient_information.html; 2008). Simple strategies such as using a large bold font, bulleted lists, short sentences, avoiding long words and technical terms, and including basic diagrams and pictures can make the text more accessible. In addition, using personal, 'everyday' language (for example, "we" to refer to the health-care provider, and "you" to refer to the patient), repeating important points, and explaining concepts in simple terms aids understanding.

Much of the research on the impact of health literacy has focused on the patient's ability to read written health information. This approach may underestimate the
Alexandra King is the Senior Editor of Nature Reviews Cardiology.

Competing interests The author declares no competing interests. 
Improving health literacy ... is a vital step towards improving outcomes 97 prevalence of poor health literacy, however, because it uses too narrow a view of 'literacy' and does not take into account listening, speaking, and writing. Being able to listen effectively and communicate questions to the physician is just as important in health literacy as reading. In addition to improving the quality and readability of written health-care information, physicians can help their patients by improving verbal communication. Recognizing and avoiding technical jargon is important, but can be difficult. Words that seem commonplace to medical professionals may be unfamiliar or confusing to patients. Asking the patient to explain their understanding of the information given to them, before they leave the office (the so-called 'teach back' approach), can also help to ensure that guidance is followed. On the other hand, the physician must also take care that the way in which they explain concepts to the patient is not patronizing. Admittedly, a delicate balance.

Health-care information, both written and verbal, should also be appropriate across age groups and cultural backgrounds. Physicians should be aware that cultural perceptions of medical conditions and treatments, and the nuances of translation from a patient's native language, can also have a role in their level of health literacy.

Adult education programs, such as the US Adult Basic Education and Literacy (ABEL) system, can be helpful if patients are identified as being poorly literate and they are willing to participate in such schemes. Within the health-care setting, interventions targeted at specific patient groups have shown some success. For example, in one study, $100 \%$ of patients with heart failure and low health literacy reported better selfmanagement and improved quality of life after they received an education booklet specifically designed for them (DeWalt, D. A. et al. Patient Educ. Couns. 55, $78-86 ; 2004)$. A more recent study showed that use of a low literacy hospital discharge tool, which could be customized to each patient, improved the accuracy with which patients with cardiovascular disease reported their medication adherence (Cordasco, K. M. et al. Am. J. Prev. Med. 37 (6 Suppl. 1), S209-S216; 2009). This finding indicates that these patients gained a greater understanding of their medication regimen through the educational intervention.

Improving health literacy and the way in which physicians communicate with patients is a vital step towards improving outcomes. The medical community must work on the basis that simply providing patients with health-care information is not sufficient, and does not necessarily guarantee their understanding.

doi:10.1038/nrcardio.2010.122 